\title{
ORBITAL INSTABILITY AND FORMING OF OUTER PART OF ASTEROIDAL BELT
}

\author{
Elena V. Alfimova \\ Shternborg State Astronomical Institute, Dept. of Celestial Mechanics, \\ Universitesky Prospect 13, Moscow, Russia
}

\section{ABSTRACT}

The or bital instability and forming of outer part of Asteroidal belt has been studied earlier in the Circular case. Here the same problem is studied in elliptic case.

\section{FORMULATION OF THE PROEL EM}

Here the case of planer restricted three-body problem will be considered. The mass of central body $P_{0}$ supposed as the unit, and the mass of perturbing body $P^{\prime}$ as $\mu \ll<$. The semi-major axis of $P^{\prime}$ will be considered as a unit of length, but a unit of time vill be selected so that the gravitational constant would become unity. For the Kepler elements of passive gravitating point we shall be using the well known terms but all elements of $P^{\prime}$ will be different.

Let us consider the first type of resonances. Thus initially we have:the condition:

$$
\left|\ell \cdot n-(\ell+n) n^{\prime}\right|<0(/ \mu) \text {, }
$$

where $\ell$ is a simple integer. Then we can introduce the Delamay anomaly as

$$
\left.S=\ell \cdot M-(\ell+1) M M^{\prime}-\psi\right)
$$

where $\psi=\omega-w^{\prime}$.

Then we carry out the expansion of perturbed function $R$ over the fast variables $M$ and 'M' and neglecting higher order terms 
in e and $e^{\prime}$. Vie shall obta in

$$
R=B_{0}+e B_{1} \cos S+e^{\prime} B_{2} \cos (S+\psi)
$$

where

$$
\begin{aligned}
B_{0} & =\mu / 2 L_{1}^{(0)}(a) \\
B_{1} & =\mu / 2\left[2(l+1) L_{1}^{(l+1)}(a)+a \frac{d}{d a} L_{1}^{(l+1)}(a)\right], \\
B_{2}= & \mu / 2\left[(2 \ell+1) L_{1}^{(l+1)}(a)+a \frac{d}{d a} L_{1}^{(l+1)}(a)\right. \\
& \left.+2 a \delta_{\ell} e^{\prime}\right], \delta_{1}=1, \delta_{\ell}=0 \text { with } \ell \neq 0,
\end{aligned}
$$

$L_{1}^{(\ell+1)}$ are the Laplace coefficients.

If we change our problem to circular problem $\left(e^{\prime}=0\right)$, we have the following integral

$$
\gamma=a\left(\ell+1-\quad \ell \sqrt{1-e^{2}}\right)^{2}
$$

At present the solution of this problem obtained with the Weierstrass-functions [2]. Some trajectories are shown in Figure 1 .

On the lower part of range takes place the separatrix and trajectories types, but on the upper some results of numerical integration.

Stationary solutions of the problem are marked as $e_{1}, e_{2}$, $e_{3}$. It's behaviour depending on the values of $\gamma$ have been shown in Figure 2 .

From the conditions of $f$ irst type trajectories there fol lows the impossibility of an approaching of a steroid and Jupiter in aphelion although that trajectories are in the instability range according to till [3]. Other trajectories in this ca se would be unstable.

Now we consider the following problem: What are the trajectories near the separatrix which change with variation to elliptic problen?

At $f$ irst let us prove the existance of ergodic layer in this case in which the turbing of orbit type are happening. The method given in [6] is being used here. 


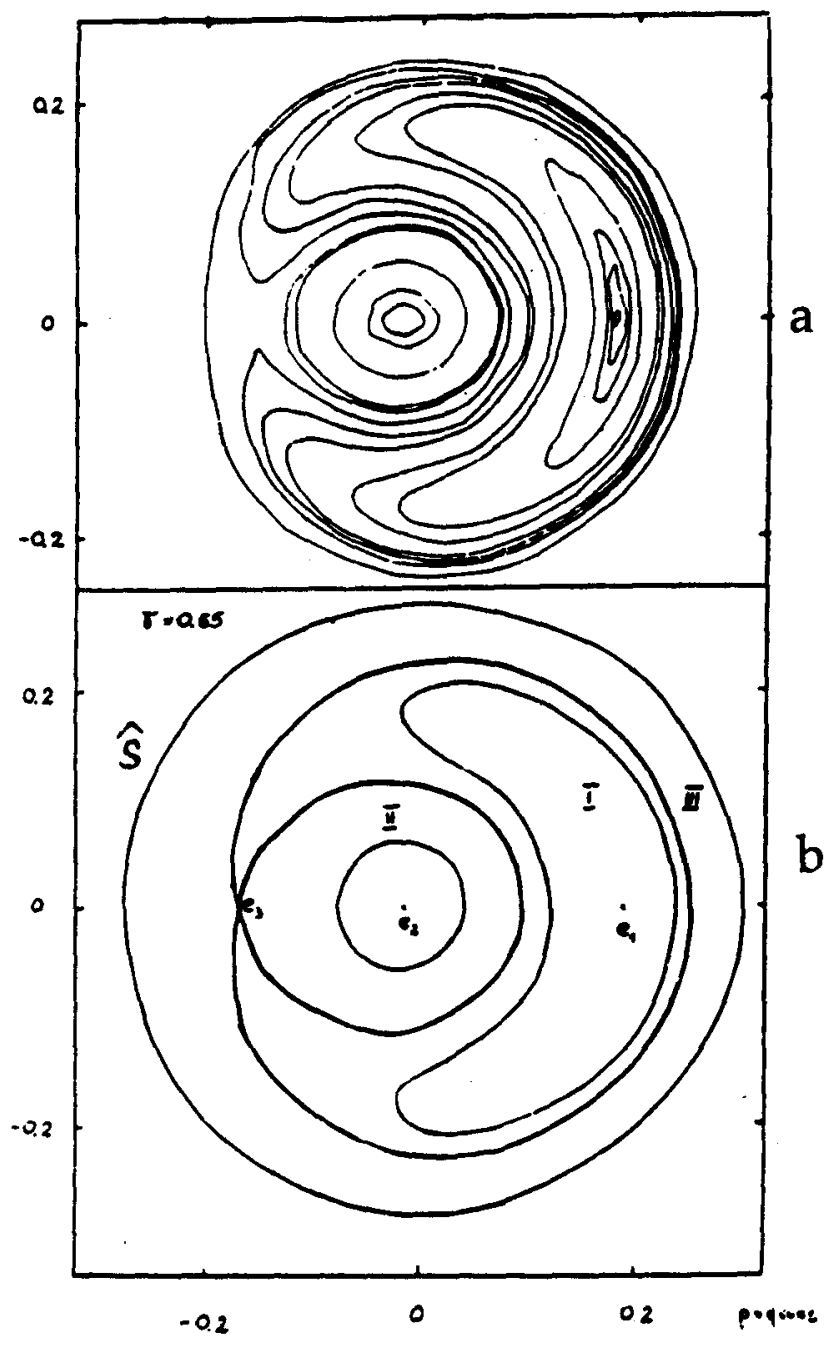

Figure 1: (a) The results of munerical integration.

(b) Separatrix $\hat{\mathbf{s}}$ and some types of trajectories.

THE PROOF OF ERGODIC LAYAR EXISTANCE

For first type layar trajectories, if we mark the mean motion $n_{1}$ of $e_{1}$, solution for 


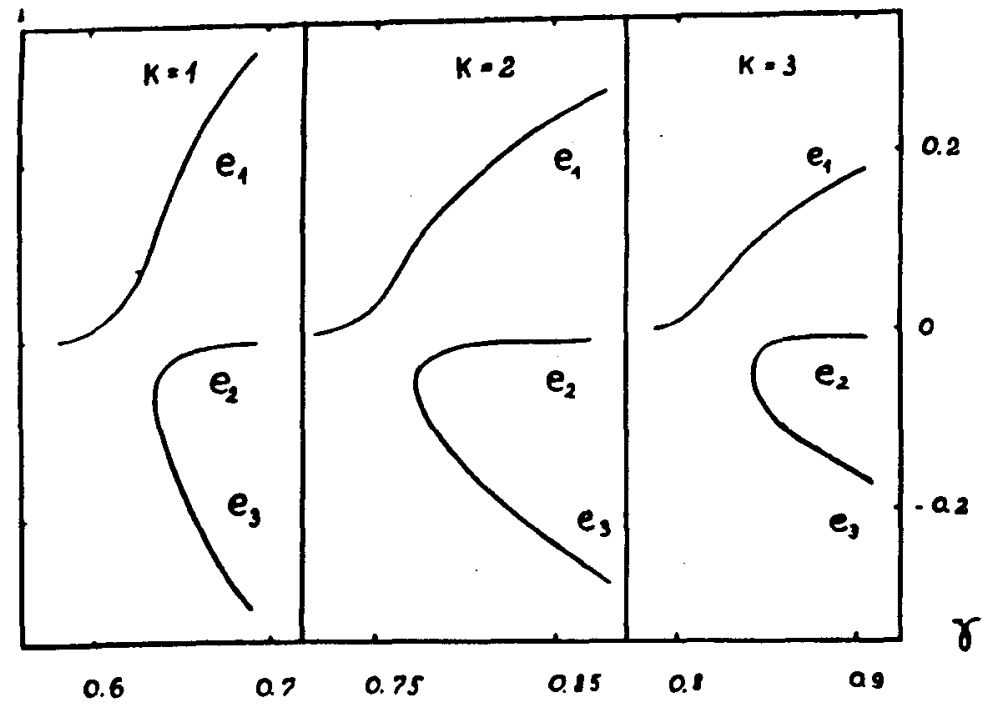

Figure 2: Behaviours of stationary solutions $e_{1}, e_{2}, e_{3}$ depend ed on the value of $\gamma$

$$
\begin{aligned}
& \Delta n=n-n_{1} \text { is } \\
& (\Delta n)^{2}=c+2 \varepsilon_{1} \text { cos } s,
\end{aligned}
$$

where $c$ is a constant, $\varepsilon_{1}=\frac{3}{2} \gamma^{-2} B_{1} e_{1}$.

Then, since $\dot{s}=\ell \Delta n$, for the second derivative we have

$$
\ddot{s}=-\varepsilon_{1} \sin s \text {, }
$$

or, with $e^{\prime} \neq 0$.

$$
\ddot{S}=-\varepsilon_{1} \sin s-\varepsilon_{2} \sin (S+\psi)
$$

Substitute the new variable $V=\tau \dot{S}$, where $\tau=\varepsilon_{1}^{-1}$, and obtain the equation (7) in the following form:

$$
\tau \dot{V}=-\sin s-\varepsilon \sin (S+\psi) \text {. }
$$

Here it is supposed $\varepsilon=\varepsilon_{2} / \varepsilon_{1}$.

We make use of Hamiltonian of unperturbed motion:

$$
\mathrm{H}=\frac{1}{2} \mathrm{~V}^{2}-\cos \mathrm{s},
$$


and bring in new variables I-action and $\psi$-fase:

$$
\begin{aligned}
& I=\frac{1}{2 \pi} \oint v a s=\frac{8}{\pi}\left[E-\left(1-K^{2}\right) K\right] \\
& \psi=\frac{\partial S}{\partial I},
\end{aligned}
$$

$$
\text { where } s=\frac{1}{2 \pi} \mathrm{ds} \mathrm{v} \text {. }
$$

Here $K, E$-are first and second type full integrals with module $\mathrm{k}=\sqrt{\frac{1+\mathrm{H}}{2}}$.

If perturbations are absent $(\varepsilon=0)$, then equations of motion would become

$$
\dot{I}=0, \quad \psi=\Omega(I),
$$

where $\Omega$ is a frequency and

$$
\Omega=\frac{1}{\tau} \frac{\mathrm{dH}}{\mathrm{dI}}=\frac{I}{2 \mathrm{~K}} \frac{1}{\tau}
$$

Mut, if perturbations are present, then

$$
\dot{I}=\frac{d I}{d H}\left(\frac{\partial H}{\partial V} \dot{V}+\frac{\partial H}{\partial S} \dot{S}\right)=-\frac{\varepsilon}{\tau^{2} \Omega} V \sin (S+\psi)
$$

Let us define the spectrum of speed $V$ in the ca se when the perturbations are a bsent $(\varepsilon=0)$. So far a $s=\tau \ell\left(n-n_{1}\right)$, then with $k>1$ we have to we ite [4]:

$$
v+2 k \operatorname{cn}(u, k) \text {, }
$$

where $u=l \tau^{-1} t$ is the new variable. Or, if we substitute the new variable $q=\exp \left(-\pi k^{\prime} / k\right)$, where $k^{\prime}=k\left(\sqrt{1-k^{2}}\right)$, a nd expand it in Fourier-series, then we have the following form:

$$
V=8 \Omega \tau \sum_{n=1}^{\infty} \frac{q^{n-\frac{1}{2}}}{1+q^{2 n-1}} \cos [(2 n-1) \Omega t]
$$

he shall consider the motion near the separatrix. In this aase we have 


$$
\begin{aligned}
& k+1, q \rightarrow 1, k \approx \frac{1}{2} \ln \frac{32}{1-H}, K^{\prime} \approx \frac{\pi}{2}, \\
& \Omega_{\tau} \approx \frac{\pi}{\ln \frac{32}{1-H}}, q \approx \exp [-\pi-\Omega \tau]
\end{aligned}
$$

Then, in the right hand side of equation (13) pick out some resonant term. Taking into account $\psi \approx$ Bt we have the following resonant condition:

$$
\tau \Omega\left(I_{N}\right)=\frac{B \tau}{N},
$$

where $N$ is an odd number and $I_{N}$ is an action by that resonance.

In the case of $B \tau \sim 1$ we have $\tau_{\Omega}\left(I_{N}\right) \sim 1 / N$, but near the separatrix $\tau \Omega \ll 1$, therefore (17) would be carried out just for $\mathrm{N} \gg 1$. Let the distance between resonances $\mathrm{N}$ and $(\mathrm{N}+2)$ be

$$
R_{N}=\Omega\left(I_{N}\right)-\Omega\left(I_{N+2}\right) \leq \frac{2}{\tau N^{2}} \sim \tau \Omega^{2}\left(I_{N}\right)
$$

Then, the stochastic condition will have the following form:

$$
\left|\frac{d \Omega\left(I_{N}\right)}{d I_{N}}\right| \delta I_{N} \gg R_{N} \simeq \tau \Omega^{2}\left(I_{N}\right),
$$

where $\delta I_{N}$ is the maximum variation of particle's action in the $\mathrm{N}$-resonance.

Assuming the variation of frequency have sufficiently snall range, we have

$$
\left|\frac{d \Omega}{d I}\left(I_{N}\right)\right| \delta I_{N} \ll \Omega\left(I_{N}\right)
$$

After integration of (13) over time-interval, such that $\succsim \tau$, in the range of $\mathrm{N}-\mathrm{res}$ nance we shall have 


$$
\delta I_{N} \sim \frac{q^{n-1 / 2}}{1+q^{l n+1}} \frac{4 \varepsilon}{\tau N\left|\frac{d \Omega}{d I}\right| \delta I_{N}}
$$

but $q \sim 1$, therefore

$$
\delta I_{N} \sim\left|\frac{2 \varepsilon \Omega\left(I_{N}\right)}{\frac{d \Omega\left(I_{N}\right)}{d I}}\right|^{1 / 2}
$$

Thus, the stochastic condition will be obtained in the fom:

$$
L=\frac{I}{R}\left|\frac{d \Omega}{d I}\right| \delta I=\sqrt{\frac{2 \varepsilon}{\tau^{2} \Omega^{3}}\left|\frac{d \Omega}{d I}\right|}>1
$$

But near the separatrix we have

$$
\left|\frac{d \Omega}{d I}\right|=\frac{\tau^{2} \Omega^{3}}{32 \pi} \exp \left(\frac{\pi}{\tau \Omega}\right)
$$

and

$$
L^{2}=\frac{\varepsilon}{16 \pi} \exp \left(\frac{\pi}{\tau \Omega}\right)>1
$$

The boundary of stochastic ca se is approximately defined from such condition: $L \lambda l$, that is within the frequency range $0 \leq \Omega \lesssim \bar{\Omega}$.

$$
\bar{\Omega}=\frac{\pi}{\tau \ln \frac{\ell 6 \pi}{\varepsilon}}
$$

From (16) we find the stochastic boundary of energy such as

$$
1-\overline{\mathrm{H}} \approx \frac{2 \varepsilon}{\pi} \sim \varepsilon
$$




\section{DEF INITION OF THE WIDTH OF SEPARATR IX SPLITTING}

Let us define the range of separatrix splitting. In the case of elliptic problem, we have

$$
\dot{\gamma}=2 \ell \sqrt{a} e^{\prime} E_{2} \sin (S+\psi)
$$

Therefore on the separatrix $s \ll \dot{\psi}$, then

$$
\gamma=\gamma_{0}+\frac{B}{B} \cos (\psi) \text {. }
$$

$U_{\Delta \gamma}$ sing the analytic form for $\beta,[4]$, we have to count the value

$$
\gamma=\gamma_{0} \pm \Delta \gamma
$$

Take that for $\ell=1, e^{\prime}=0.048, e=0,14, \mu=1 / 1047$ and for the other conditions corresponding to [1].

We observe from $F$ igure 3. that as $\gamma$ changes it's value from 0.64 to 0.652 , that to 0.18 , the right boundary of projecting will be changed from 0,2 to 0.26 .

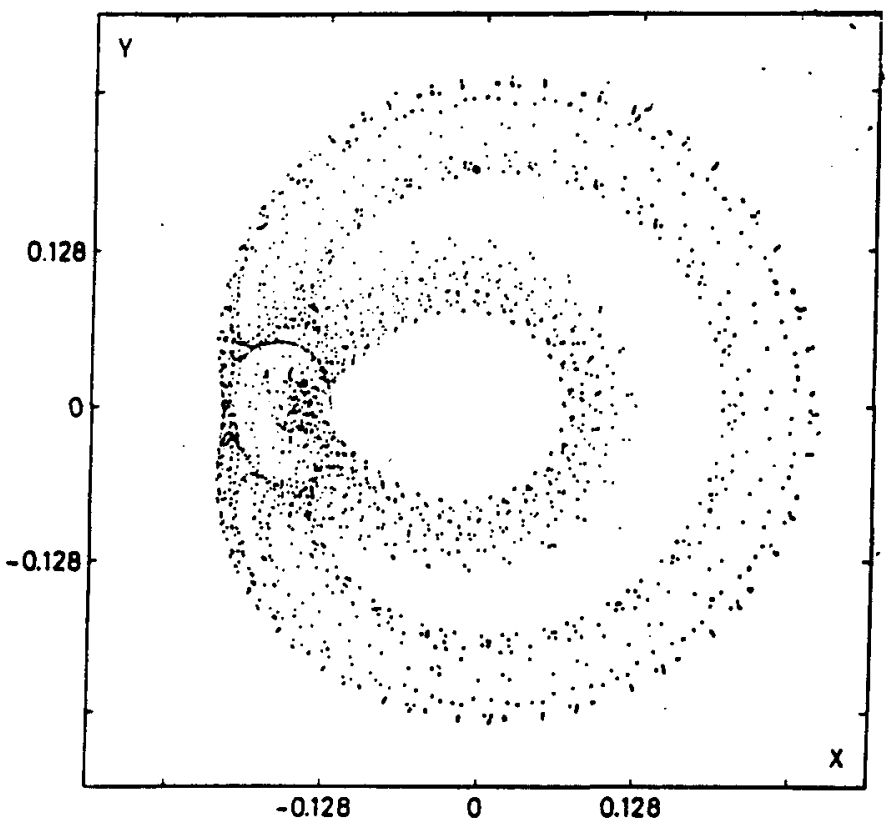

Figure 3: Variation of $e_{3}$-solution depending on the variation of $0.64 \leq \gamma \leq 0.652 . X=e_{3} \cos s_{1} Y=e_{3} \sin S$. 
Analogous change of $K=\sqrt{\gamma}$ are shown in Figure 4 . Our future plan is to apply this method for a steroids of Gilda's group and in particular, for a steroid no.334, who se motion have been investigated by shubart [5].

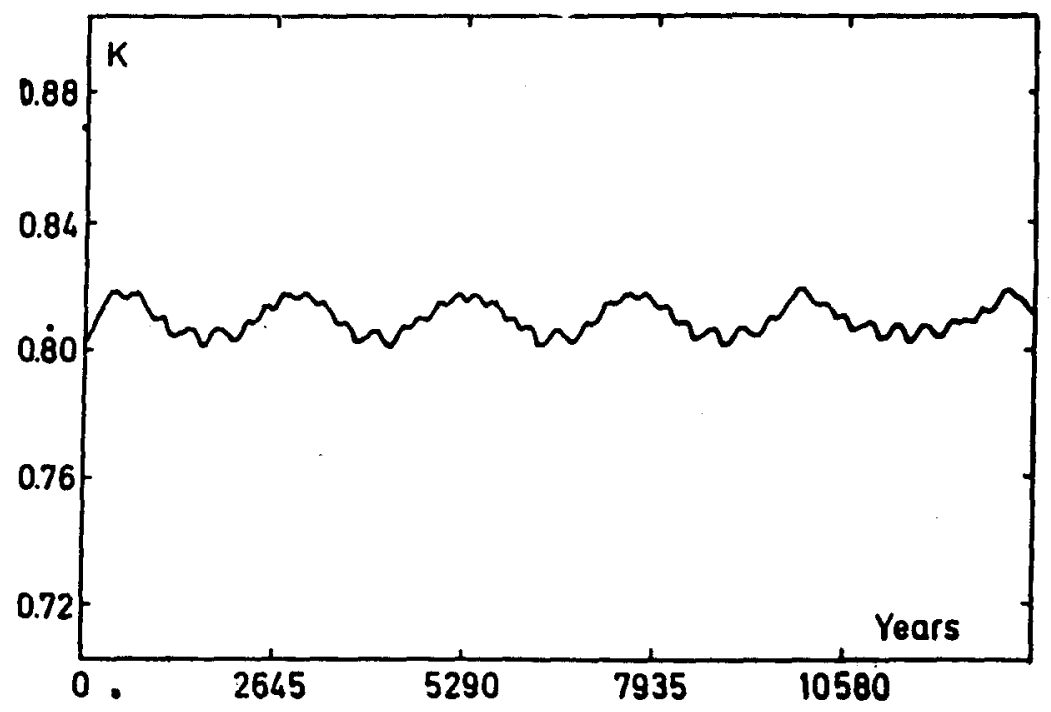

Figure 4: Variation of $K=\sqrt{\gamma}$ according to $0.64 \leq \gamma \leq 0.652$.

\section{REF ERENCES}

[1] C.Froeschle, H. School, Astronomy and Astrophysics, V 51 , 1977 .

[2] I.A. Gera simov, Astronomic hesky Z hurnal, V .63,1986.

[3] I.A. Geka simov, N.A. Solovaya, Meetino of Uppsala Univer sity, June 12-16,1989.

[4] I.A. Geka simov, N.V.Kiru shenkova, Ko sm ic heskie issledovanja, V.27, No.5, 1989 .

[5] J.Schubrt, Celestial Mechanics, V.43, 1988.

[6] N.A. Zaslavskyi, Stochastic instability in the nonlinear processes, Hoscow, 1970 . 